\title{
Honoré de Balzac, Correspondance I, (1809-1835)
}

\section{Raffaele De Cesare}

\section{(2) OpenEdition}

\section{Journals}

\section{Edizione digitale}

URL: http://journals.openedition.org/studifrancesi/27128

DOI: 10.4000/studifrancesi.27128

ISSN: 2427-5856

\section{Editore}

Rosenberg \& Sellier

\section{Edizione cartacea}

Data di pubblicazione: 31 décembre 2006

Paginazione: $568-569$

ISSN: 0039-2944

\section{Notizia bibliografica digitale}

Raffaele De Cesare, «Honoré de Balzac, Correspondance I, (1809-1835)», Studi Francesi [Online], 150 (L I III) | 2006, online dal 30 novembre 2015, consultato il 08 novembre 2020. URL : http://

journals.openedition.org/studifrancesi/27128; DOI : https://doi.org/10.4000/studifrancesi.27128

\section{(c) (i) (9)}

Studi Francesi è distribuita con Licenza Creative Commons Attribuzione - Non commerciale - Non opere derivate 4.0 Internazionale. 
Honoré de Balzac, Correspondance I, (1809-1835), édition établie, présentée et annotée par Roger Pierrot et Hervé Yon, Paris, Gallimard, "Bibliothèque de la Pleiade", 2006, pp. 1604.

La prima edizione, criticamente accertata, del volume che dà inizio alla Correspondance di Balzac (contenente le lettere dal 1809 al 1832) a cura di Roger Pierrot, fu pubblicata a Parigi, nella serie dei Classiques Garnier, nel 1960. Seguì, due anni dopo, l'edizione del secondo volume che raccoglieva le lettere appartenenti al triennio 1833-1835. Con cadenza regolare fu continuata infine, sino al 1969 , con altri tre tomi, comprensivi delle missive dello scrittore, dal 1836 alle ultime (1850), corredate da un supplemento di quelle rintracciate durante e dopo la pubblicazione dell'intero epistolario.

L'opera fu subito accolta con grande favore dal pubblico che vedeva così colmana una grande lacuna negli studi balzacchiani; e le ricerche laboriose, esemplari per erudizione e per vigilnza critica di Roger Pierrot conobbero il riconoscimento che era ad esso dovuto grazie al magistrale impegno che vi era profuso ${ }^{1}$.

A distanza di mezzo secolo (l'uscita del quinto volume è, come si è già accennato, del 1969), l'opera riappare ora alla luce nelle sue due prime parti, rivista, corretta, aumentata, assistita dalla collaborazione di Hervé YON, che ha contribuito nell'allestimento di essa al lavoro di Pierrot.

Pur trattandosi di una riedizione, questo "corpus" epistolare di circa tremila lettere (che, per gli anni 1809-1832, è folto di quasi duecento nuovi documenti) merita un intervento recensorio che torni, sia pure sommariamente, ad illustrare i meriti del principale curatore di esso, oggi uno dei migliori specialisti di Balzac, e del più giovane e non meno benemerito collaboratore.

$\mathrm{Ma}$, anzitutto, bisogna spendere una parola di plauso nei riguardi della casa editrice Gallimard che ha inserito nella sua ormai famosa "Bibliothèque de la Pléiade" anche le pagine epistolografiche ed i documenti ad esse connessi del grande romanziere. La "Pléiade" annoverava già il testo della Comédie bumaine sia in una prima edizione curata da Marcel Bouteron, sia in una successiva (1976-1981), ben più completa, preparata, sotto la direzione di Pierre-Georges Castex, provvista di un apparato di varianti testuali ed arricchita da introduzioni, note e commenti critici di un gruppo di eminenti studiosi. Ad essa affiancava i primi due volumi delle Oeuvres diverses (1990-1996: un terzo è atteso fra breve) a cura diRoland Chollet e del compianto René Guise; un suggestivo Album Balzac (1962), repertorio iconografico riunito da Jean Ducourneau. Mancano ancora, è vero, il Théatre e le
Lettres à l'Etrangére (ma dell'uno esiste già l'edizione Guise per i tipi dei "Bibliophiles de l'Originale" (1969-1973) e, delle altre, le due edizioni degli stessi "Bibliophiles de L'Originale" (19671969) e del Laffont (1990) dovute entrambe a Roger Pierrot). Si può dire perciò che la collana di Gallimard raduni ormai la massima parte dell'intera opera balzacchiana; e ce se ne può attendere al più presto il completamento: coronamento che permetterà ad un pubblico più vasto di consultare rapidamente, unitariamente e fiduciosamente un "tutto Balzac", che agevolerà cioè di seguire nel modo migliore, in ogni sua tappa, il cammino vulcanico, intellettuale e psicologico percorso dal grande scrittore.

Premesse queste doverose parole di elogio l'iniziativa di una casa editrice attenta a favorire le esigenze di un pubblico europeo, come quella di Gallimard, è opportuno insistere nel segnalare il fatto che questa seconda edizione della Correspondance non è, come del resto abbiamo già detto, una ristampa della prima, ma una vera e propria nuova "mise sur le métier". Essa corregge le rare sviste di lettura (inevitabili nell'allestimento di ogni epistolario), elimina le incertezze di datazione, identifica le sigle di alcuni corrispondenti, sgombra insomma molti dubbi rimasti insoluti nell'edizione precedente e, facendo tesoro dei recenti ritrovamenti e delle più approfondite precisazioni bibliografiche, approda così a una più sicura collocazione delle testimonianze epistolari distribuite ora nel loro più convincente contesto, e ad una illustrazione più completa delle vicende umane del loro autore.

È per tale insieme di fatti che si giustifica - ripetiamo - il nuovo richiamo bibliografico che intendiamo appunto delineare.

Esso ha l'intento di rendere edotto il lettore di quanto l'opera di R. Pierrot arricchisca e renda somigliante alla realtà il ritratto di Balzac offerto all'ammirazione di quanti vedono in lui uno dei massimi romanzieri (forse il massimo) dell'Ottocento francese, un secolo incomparabile per una fioritura narrativa che si specchia - è tutto dire! - in uno Stendhal, in un Mérimée ed in un Flaubert.

Procedendo ora, a rapidi tratti, ad una analisi particolare di questo primo volume, segnaliamo le principali novità che emergono dalla lettura dell'opera.

Rileviamo anzitutto la presenza di alcune lettere nuovamente scoperte o collazionate per 
intero sugli autografi, a Zulma Carraud del 4 febbraio 1831 (p. 327); ad Alfred de Vigny del giugno o luglio 1831 (p. 368); a Charles de Bernard del 3 ottobre 1831 (?) (p. 406); a Ferdinand Bellizard, editore della "Revue étrangère" di San Pitroburgo, dell'aprile 1833 (pp. 770771); ad Alphonse Royer, direttore dell'"Europe littéraire" del luglio e dell'agosto 1833 (?) (pp. 814-815); al barone Gérard del 28 marzo 1835 (pp. 1072-1073).

Rileviamo poi l'inserimento di numerose lettere, escluse dalla prima edizione o apparse dopo di essa, di familiari, di amici, di conoscenti, di ammiratori e di ammiratrici (di queste v'è una vera e propria pioggia...) fra le quali risaltano, a diverso titolo, il frammento di una lettera della marchesa de Castries del 7 giugno 1832 (p. 537) che svela un aspetto della dolce-amara relazione sentimentale della nobildonna e dello scrittore; la lettera di una tale contessa d'Escarbagnas des antiques" provinciale, che sconsacra la memoria di Molière, protesta contro Montalembert e - confondendo date e nomi - ritiene dapprima il corrispondente discendente dalla famiglia di Guez de Balzac (e ne fa un proprio parente...), poi, avvicinandosi alle pretese di Honoré, ma non alla realtà, lo assimila alla casata dei Balzac d'Entragues; il testo integrale della lettera del marchese Astolphe de Custine del marzo 1835 (pp. 1071-1072) relativa al Père Goriot; la lettera di Etienne Geoffroy de Saint-Hilaire del 4 luglio 1835 (?) (p. 1107) invocante un abboccamento («j'ai faim de vous voir»).

Ambedue questi gruppi di lettere aiutano il lettore a ricostruire anche in certi minimi det- tagli, la vita prodigiosa intessuta di miserie e di splendori, di genialità e di sregolatezza, di un uomo perennemente diviso fra manifestazioni fanciullesche di vanità, tentazioni di dispersione mondana e di snobismo, ansia di solitario raccoglimento spirituale, austera dedizione ad una concezione irreprensibile dell'arte, costantemente tormentato dalla furia rabbiosa dell'invenzione e dall'incubo della perfezione stilistica, generoso ed avido (e si riflette all'accortezza commerciale - spesso astuzia bella e buona - dei suoi contratti editoriali) quale fu quella, complessa e contradditoria di Balzac. E contribuiscono a valutare in una più giusta misura in quale atmosfera si sia svolta quella impressionante "manufacture d'idées" che distingue la Comédie bumaine da ogni altra opera ciclica della narrativa europea ottocentesca.

Insomma, fin dalla chiusura di questo primo volume, il lettore può rendersi conto di quale grande servizio Roger Pierrot, coronando una vita intera di studioso dedicata con passione a competenza a Balzac, abbia reso alla memoria del grande romanziere. Che ognuno di noi possa degnatamente manifestargli sincera gratitudine

[RAFFAELE DE CESARE]

${ }^{1}$ Mi permetto ricordare qui che della pubblicazione del primo volume, nel 1960, mi occupai anch'io in una recensione apparsa in «Rivista di letterature moderne e comparate», vol. XIV, 33, settembre 1961, pp. 206-210. 